Artículo Original. El rol de la resiliencia en la asociación entre la actividad física deportiva y aspectos académicos en escolares. Vol. 5, n. ${ }^{\circ}$; p. 513-531, septiembre 2019. A Coruña. España ISSN 2386-8333.

\title{
El rol de la resiliencia en la asociación entre la actividad física deportiva y aspectos académicos en escolares
}

The role of resilience in the association between sports physical activity and academic aspects in schoolchildren

Irwin Andrés Ramírez Granizo ${ }^{1}$; Jose Manuel Alonso Vargas ${ }^{1}$, Silvia San Román Mata²; José Luis Ubago Jiménez ${ }^{1}$; Asunción Martínez Martínez ${ }^{3}$; María Sánchez Zafra ${ }^{4}$

${ }^{1}$ Departamento de Didáctica de la Expresión Musical, Plástica y Corporal. Universidad de Granada.

${ }^{2}$ Departamento de Métodos de Investigación y Diagnóstico en Educación. Universidad de Granada.

${ }^{3}$ Universidad de Granada.

${ }^{4}$ Departamento de Didáctica de la Expresión Musical, Plástica y Corporal. Universidad de Jaén

Autor de correspondencia: María Sánchez

Contacto: mszafra@ujaen.es

Cronograma editorial: Artículo recibido: 21/07/2019 Aceptado: 26/08/2019 Publicado: 01/09/2019 DOI: https://doi.org/10.17979/sportis.2019.5.3.5603

Para citar este artículo utilice la siguiente referencia: Ramírez, I.A.; Alonso, J.M.; San Román, S.; Ubago, J.L.; Martínez, A.; Sánchez, M. ${ }^{a}$ (2019). El rol de la resiliencia en la asociación entre la actividad física deportiva y aspectos académicos en escolares. Sportis Sci J, 5 (3), 484- 531 


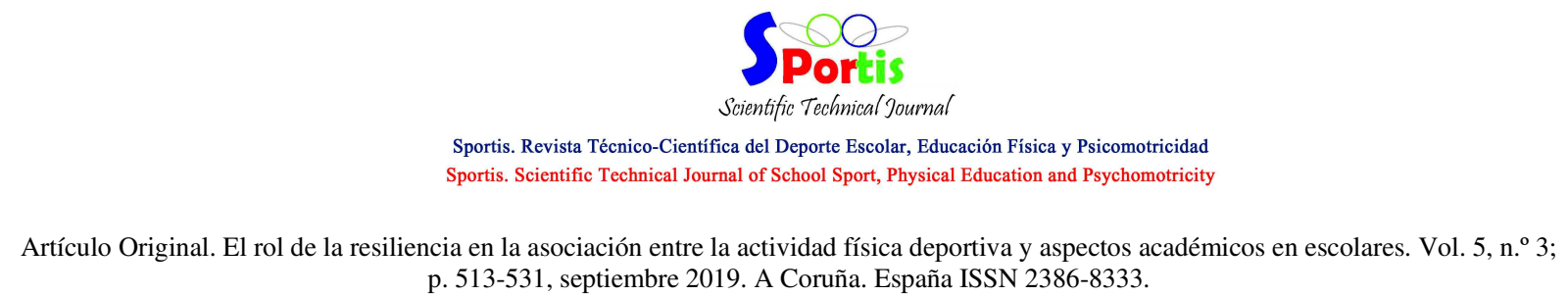
p. 513-531, septiembre 2019. A Coruña. España ISSN 2386-8333.

\title{
Resumen
}

El concepto de resiliencia se define como el conjunto de cualidades intrínsecas que un individuo posee para poder superar situaciones adversas. En los últimos años se ha demostrado que esta capacidad puede ser trabajada y mejorada a partir de la práctica de actividad física y el deporte, siendo un campo de investigación de sumo interés. Este estudio tiene como objetivo principal analizar la relación existente entre los niveles de resiliencia, la práctica de actividad física y variables académicas (haber repetido o no), en una muestra de escolares. Para ello se realizó un estudio descriptivo de corte transversal en una muestra compuesta por 320 escolares $(11,25 \pm 0,53)$, que cursaban tercer ciclo de Primaria. Como principales resultados, la dimensión que muestra un mayor nivel de resiliencia es la de persistencia, seguida de control bajo presión y adaptabilidad donde en ésta última el género femenino destaco por encima del masculino. Asimismo, destaca que aquellos sujetos que realizaban mayor actividad física presentaban características más resilientes que los que no hacían ejercicio físico. En la variable académica, hay una asociación entre los participantes más resilientes y no haber repetido curso. A partir de dicho estudio se revela como practicar actividad física ayuda a incrementar la capacidad resiliente debido a que su práctica ayuda a aceptar positivamente los cambios y esto a su vez puede influir en el rendimiento académico.

\section{Palabras clave}

Resiliencia; actividad física; rendimiento académico; escolares.

\begin{abstract}
The concept of resilience is defined as the set of intrinsic qualities that an individual possesses in order to overcome adverse situations. In recent years it has been demonstrated that this ability can be worked and improved from the practice of physical activity and sport, being a field of research of great interest. The main objective of this study is to analyse the relationship between levels of resilience, physical activity practice and academic variables (having repeated or not) in a sample of schoolchildren. For this purpose, a descriptive crosssectional study was carried out on a sample of 320 schoolchildren $(11.25 \pm 0.53)$, who were in the third cycle of Primary school. As main results, the dimension that shows the highest level of resilience is persistence, followed by control under pressure and adaptability where in the latter the female gender stands out above the male. It also points out that those subjects who did more physical activity had more resilient characteristics than those who did not do physical exercise. In the academic variable, there is an association between the most resilient participants and not having repeated a course. From this study it is revealed how practicing physical activity helps to increase resilience because its practice helps to accept changes positively and this in turn can influence academic performance.
\end{abstract}

\section{Keywords}

Resilience; physical activity; academic achievement; children.

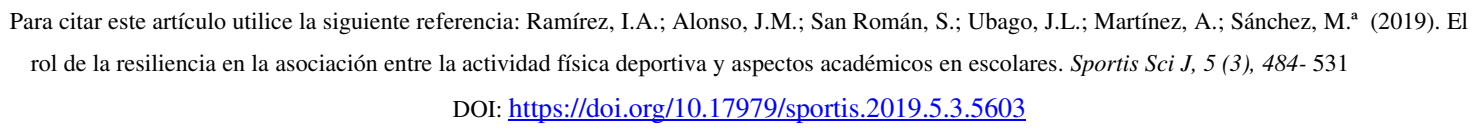


Artículo Original. El rol de la resiliencia en la asociación entre la actividad física deportiva y aspectos académicos en escolares. Vol. 5, n. ${ }^{\text {o }}$ 3; p. 513-531, septiembre 2019. A Coruña. España ISSN 2386-8333.
\end{abstract}

\title{
1. Introducción
}

Cuando se habla sobre resiliencia se debe entender como el elemento o la capacidad que tiene una persona para poder superar situaciones negativas y poder promover el proceso de superar aquellos efectos negativos a los cuales unos se puede encontrar expuesto (Zurita, Castro, Linares y Chacón, 2017). El ser humano al estar expuesto a experiencias de interacción y relación con diferentes contextos a lo largo de toda su vida, tiene que adaptarse y cada individuo puede reaccionar de una manera u otra a situaciones adversas, pudiendo así superarlas o no. Los sujetos que lo consiguen muestran lo que se llama una actitud resiliente o un alto grado de resiliencia que les permite adaptarse y sobreponerse a estas experiencias (Broche, Diago y Herrera, 2012). Por ello, una definición de resiliencia la da CabanyesTruffino (2010), haciendo referencia al conjunto de cualidades del sujeto que configuran la habilidad de una persona a sobreponerse o superar situaciones agobiantes o difíciles.

Anteriormente Grobert (1995) describió este término como la capacidad que tiene el ser humano para hacer frente a las adversidades que la vida presenta, poder superarlas y así aprender y mejorar de ellas, siendo esto también definido anteriormente por Rutter (1993) como aquel mecanismo protector que permite al individuo poder salir de una situación difícil o de una adversidad, fortalecido. Por lo tanto, es importante estudiarla ya que es importante potenciar esos aspectos que permitan superar las situaciones adversas y desfavorables (Bretón, Zurita y Cepero, 2017; Ortín-Montero et al., 2013). Pero ha de saberse que la resiliencia no es un aspecto heredado por el sujeto, sino que éste se adquiere a medida que este se pasa por diferentes situaciones o etapas de su vida, siendo imprescindible su desarrollo y trabajo en edades tempranas

Se ha mostrado la relación positiva entre la capacidad resiliente y la práctica de actividad física (Castro-Sánchez, Chacón-Cuberos, Zurita y Espejo, 2016; Ramírez-Granizo y CastroSánchez, 2018). En la sociedad de hoy en día la práctica de actividad física es un tema primordial y prioritario, debido a su especial relevancia en la salud de las personas, Espejo, Zurita, Chacón, Castro, Martínez-Martínez y Pérez-Cortés (2018) señalaron al concepto salud

Para citar este artículo utilice la siguiente referencia: Ramírez, I.A.; Alonso, J.M.; San Román, S.; Ubago, J.L.; Martínez, A.; Sánchez, M. ${ }^{a}$ (2019). El rol de la resiliencia en la asociación entre la actividad física deportiva y aspectos académicos en escolares. Sportis Sci J, 5 (3), 484- 531 


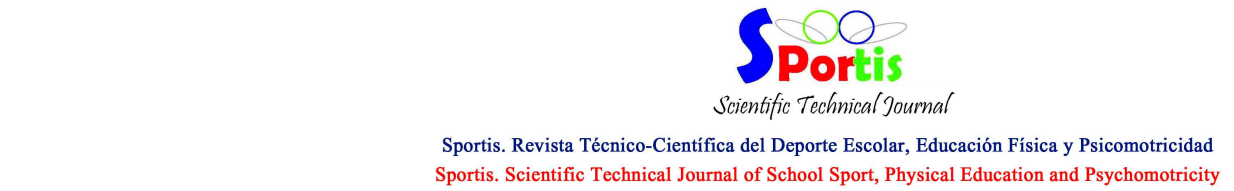

Artículo Original. El rol de la resiliencia en la asociación entre la actividad física deportiva y aspectos académicos en escolares. Vol. 5, n. ${ }^{\text {o }}$ 3; p. 513-531, septiembre 2019. A Coruña. España ISSN 2386-8333.

como aquel estado de bienestar completo, tanto a nivel físico, social como psíquico y no solamente a falta de enfermedad, por esto muchos son los estudios que trabajan los beneficios de un buen nivel de ejercicio físico sobre la salud de los sujetos (Jiménez-Beatty, Graupera, Martínez del Castillo, Martín y Campos, 2007; Zurita et al., 2009; Zurita, Castro, Linares y Chacón, 2017). Los beneficios de una práctica de actividad física se ven durante todas las etapas de la vida en especial durante la infancia. Ésta ha sido vista como un factor protector frente a numerosas enfermedades cardiovasculares, diabetes, salud mental y obesidad (Branscum y Crowson, 2017; Dentro et al., 2014). Entre todos ellos Bailey (2016) habla sobre los profundos efectos durante la infancia, destacando el crecimiento muscular y esquelético, el desarrollo de las habilidades perceptivas motrices y la capacidad de calibrar las reacciones emocionales ante situaciones impredecibles.

Siguiendo esta línea, autores como Ramírez-Granizo, Valdivia-Moral, Zurita-Ortega y Ubago-Jiménez (2018) o Subirats, Subirats y Soteras (2012), muestran también los diversos beneficios que tiene una práctica moderada de actividad física para el estado de salud de las personas, a pesar de que en ocasiones pueda provocar lesiones en el aparato locomotor o incluso riesgos para el sistema cardiovascular, mostrando que una realización sistemática de ejercicio físico supera a esto (Zurita, Espejo, Cofre, Martínez, Castro y Chacón, 2017). Asimismo, otro de los beneficios se puede ver reflejado en investigaciones como las de Kyle, Hernández, Reigal y Morales (2016), donde existen valoraciones positivas entre el autoconcepto general y sus dimensiones y la práctica de actividad física. En España son escasos los estudios sobre Resiliencia, aunque cabe destacar los realizados por De la Vega, Rivera y Ruiz (2011), trabajados en el ámbito del atletismo, el de Ruiz, De la Vega, Poveda, Rosado y Serpa (2012) en relación al fútbol o el de Reche, Tutte y Ortín (2014) sobre judo. En relación a esto y la carencia de estudios que relacionen la Resiliencia y los aspectos académicos se ha planteado este estudio ya que al tener en cuenta los profesionales de la enseñanza, profesores y maestros de educación física o psicólogos son las personas encargadas de realizar las intervenciones necesarias y de poder detectar cualquier tipo de problema a nivel psicológico o físico por lo cual este estudio intenta dar un paso más y así

Para citar este artículo utilice la siguiente referencia: Ramírez, I.A.; Alonso, J.M.; San Román, S.; Ubago, J.L.; Martínez, A.; Sánchez, M. ${ }^{a}$ (2019). El rol de la resiliencia en la asociación entre la actividad física deportiva y aspectos académicos en escolares. Sportis Sci J, 5 (3), 484 - 531 DOI: https://doi.org/10.17979/sportis.2019.5.3.5603 


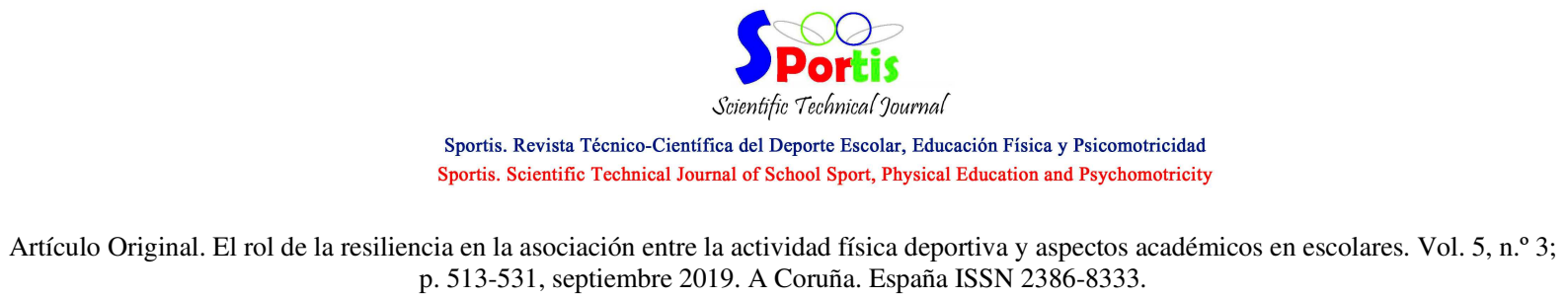
p. 513-531, septiembre 2019. A Coruña. España ISSN 2386-8333.

estudiar de manera más explícita la asociación positiva entre tener un alto nivel de resiliencia con la práctica de actividad física en niños y niñas de primaria.

Teniendo en cuenta estos antecedentes, se plantearon los siguientes objetivos:

a) determinar y analizar las propiedades psicométricas del CD-RISC en una población de escolares.

b) analizar las relaciones existentes entre los niveles de resiliencia y las variables físicodeportivas.

c) analizar las relaciones existentes entre las variables académicas (repetidor o no) y las dimensiones psicosociales (resiliencia)

\section{Material y Método}

\section{Diseño y participantes}

Participaron en este estudio de carácter descriptivo y de corte transversal, de diseño ex post facto y de grupo simple un total de 320 escolares de Primaria. La selección de los participantes se realizó a través de un muestreo aleatorio por conglomerados, considerando los escolares matriculados en tercer ciclo de Educación Primaria según los centros educativos que dieron la aceptación a formar parte del estudio. Con una edad comprendida entre los 10 y 12 años $(\mathrm{M}=11.25$ años; $\mathrm{DE}=0.53)$, representado 166 al género femenino $(51.9 \%)$ y 154 al masculino $(48,1 \%)$.

\section{Variables e Instrumentos}

Se emplearon diversos instrumentos validados para evaluar la capacidad resiliente y sus dimensiones y la actividad físico-deportiva.

- Cuestionario Sociodemográfico. Se elaboró un cuestionario de elaboración propia específico para el registro de las variables deportivas (número de horas de actividad física fuera del recinto escolar, actividad física parental, estar o no federado) al igual que las variables académicas (si ha repetido o no).

- Escala de resiliencia de Connor-Davidson (CD-RISC) (Connor y Davidson, 2003) para determinar los niveles resilientes de cada individuo. Consta de 25

\footnotetext{
Para citar este artículo utilice la siguiente referencia: Ramírez, I.A.; Alonso, J.M.; San Román, S.; Ubago, J.L.; Martínez, A.; Sánchez, M. ${ }^{a}$ (2019). El rol de la resiliencia en la asociación entre la actividad física deportiva y aspectos académicos en escolares. Sportis Sci J, 5 (3), 484- 531 DOI: https://doi.org/10.17979/sportis.2019.5.3.5603
} 
ítems; donde cada persona debe señalar hasta qué punto cada afirmación ha sido verdadera en su caso durante los últimos 30 días. Para ello, se utiliza una escala tipo Likert de 4 puntos donde el valor 0 significa "no ha sido verdadera en absoluto" y el 4 "casi siempre verdadera". La factoraliedad de los ítems permite que este instrumento configure 5 dimensiones: competencia personal y tenacidad, confianza y tolerancia a la adversidad, aceptación positiva del cambio, Control e influencia espiritual. Se obtuvo un alpha de Cronbach de $\alpha=$ .859. La resiliencia se considera alta si se supera los 75 puntos al hacer el sumatorio y baja si esta cifra es inferior (Chacón-Cuberos et al., 2016).
\end{abstract}

\title{
Procedimiento
}

Primero de todo, se procedió a solicitar la colaboración de los centros escolares a través de una carta informativa elaborada desde el Área de Corporal de la Universidad de Granada. Concertando una cita con el/la director/a de los centros, a los cuales se les entregó una copia del cuestionario para su evaluación y su correspondiente carta informativa para los padres donde se detalla los fines y la naturaleza del estudio a realizar, solicitando así su consentimiento informado para llevar a cabo la investigación.

Una vez aceptada la propuesta por el director y los tutores de los menores, se estableció un horario para la realización de dichos cuestionarios, realizándose en horario lectivo en la clase de educación física. El proceso se produjo sin ninguna incidencia, siempre estando en presencia uno o dos de los investigadores para la correcta aplicación del cuestionario y las posibles dudas que se planteen. Una vez finalizado el estudio, se entregó un informe con los resultados a los centros. Para la confidencialidad de los participantes, se aseguró el anonimato a todos los escolares, los cuales participaron de manera voluntaria y respetando el acuerdo de ética de Investigación de Helsinki. Siendo aprobado de igual manera por el comité ético de la Universidad de Granada. Del total de cuestionarios se invalidaron 53 por encontrarse mal cumplimentados.

\section{Análisis de los Datos}

El análisis estadístico se ha realizado a través del software IBM SPSS® 22.0. Para los descriptivos básicos se emplearon frecuencias y medias, mientras que para el estudio de

Para citar este artículo utilice la siguiente referencia: Ramírez, I.A.; Alonso, J.M.; San Román, S.; Ubago, J.L.; Martínez, A.; Sánchez, M. ${ }^{a}$ (2019). El rol de la resiliencia en la asociación entre la actividad física deportiva y aspectos académicos en escolares. Sportis Sci J, 5 (3), 484 - 531 
Artículo Original. El rol de la resiliencia en la asociación entre la actividad física deportiva y aspectos académicos en escolares. Vol. 5, n. ${ }^{\text {o }}$ 3; p. 513-531, septiembre 2019. A Coruña. España ISSN 2386-8333.

relaciones entre variables se ha utilizado tablas de contingencia, T de Student y Anova de un factor, dependiendo de la naturaleza de las variables. Para medir la significatividad de las variables estudiadas se utilizó el chi cuadrado de Pearson. La normalidad de los datos se realizó mediante el test de Kolmogorov-Smirnov, utilizando la corrección de Lillieforts y la homocedasticidad a través del test de Levene. La fiabilidad interna de los instrumentos empleados fue valorada mediante el coeficiente alfa de Cronbach, fijando el Índice de Confiabilidad en el 95,5\%.

\section{Resultados}

Tomando como referencia los datos de la tabla 1, la muestra estudiada presentaba proporciones similares por género, siendo el $48,1 \%(n=154)$ varones y el $51,9 \% \quad(n=166)$ féminas. El 70,6\% (n=226) de los escolares practicaban más de tres horas de actividad física fuera del centro escolar, el 29,4\% ( $n=94)$ no practicaban ejercicio físico, el 55\% ( $n=176)$ no estaba federado en ninguna disciplina deportiva y únicamente el 5\% $(\mathrm{n}=16)$ había repetido curso.

Tabla 1. Características de la muestra

\begin{tabular}{|c|c|c|c|c|c|}
\hline \multicolumn{2}{|c|}{ Género } & \multicolumn{4}{|c|}{ Repetidor } \\
\hline & Frecuencia & $\%$ & & Frecuencia & $\%$ \\
\hline Masculino & $\mathrm{n}=154$ & 48.1 & $\mathbf{S i}$ & $\mathrm{n}=16$ & 5 \\
\hline Femenino & $n=166$ & 51.9 & No & $\mathrm{n}=304$ & 95 \\
\hline Total & 320 & & Total & $\mathrm{n}=320$ & \\
\hline \multicolumn{2}{|c|}{ Actividad física $<3 \mathrm{~h}$} & \multicolumn{4}{|c|}{ Estar federado } \\
\hline & Frecuencia & $\%$ & & Frecuencia & $\%$ \\
\hline $\mathbf{S i}$ & $n=226$ & 70.6 & $\mathbf{S i}$ & $\mathrm{n}=114$ & $45 \%$ \\
\hline No & $\mathrm{n}=94$ & 29.4 & No & $\mathrm{n}=176$ & $55 \%$ \\
\hline Total & $\mathrm{n}=320$ & & Total & $\mathrm{n}=320$ & \\
\hline
\end{tabular}

Para citar este artículo utilice la siguiente referencia: Ramírez, I.A.; Alonso, J.M.; San Román, S.; Ubago, J.L.; Martínez, A.; Sánchez, M. ${ }^{a}$ (2019). El rol de la resiliencia en la asociación entre la actividad física deportiva y aspectos académicos en escolares. Sportis Sci J, 5 (3), 484- 531 
Artículo Original. El rol de la resiliencia en la asociación entre la actividad física deportiva y aspectos académicos en escolares. Vol. 5, n. ${ }^{\text {o }}$ 3; p. 513-531, septiembre 2019. A Coruña. España ISSN 2386-8333.

En cuanto a las dimensiones de Resiliencia, la persistencia obtuvo valores medios de 22,84 , siendo esta la mejor valorada entre todas. Las siguientes categorías obtuvieron valores similares con el control bajo presión $(\mathrm{M}=19.80)$ y la adaptabilidad $(\mathrm{M}=14.90)$, así las dimensiones de control y propósito y la espiritualidad fueron las que menor puntuación obtuvieron tal y como se puede ver en la Tabla 2.

Tabla 2. Valores medio de Resiliencia

\begin{tabular}{lll}
\hline \multicolumn{3}{c}{ Resiliencia } \\
\hline & Media & DT \\
Persistencia & 22.84 & 3.926 \\
Control Bajo Presión & 19.80 & 4.294 \\
Adaptabilidad & 14.90 & 2.749 \\
Control y Propósito & 9.70 & 1.942 \\
Espiritualidad & 5.84 & 1.322 \\
\hline
\end{tabular}

La tabla 3 muestra las diferencias por género en las variables académicas y físico deportivas. Se observa cómo el 62,5\% de los federados corresponde al género masculino y el $37,5 \%$ al femenino. En cuanto a la práctica física mayor a 3 horas, tanto niñas 49,56\% $(n=112)$ como niños 50,44\% $(n=114)$ obtuvieron valores similares y en la categoría de estar federado el porcentaje de mujeres es menor $43,05 \%(n=62)$ al de los hombres $56.9 \%(n=82)$. 
Artículo Original. El rol de la resiliencia en la asociación entre la actividad física deportiva y aspectos académicos en escolares. Vol. 5, n. ${ }^{\text {o }}$ 3; p. 513-531, septiembre 2019. A Coruña. España ISSN 2386-8333.

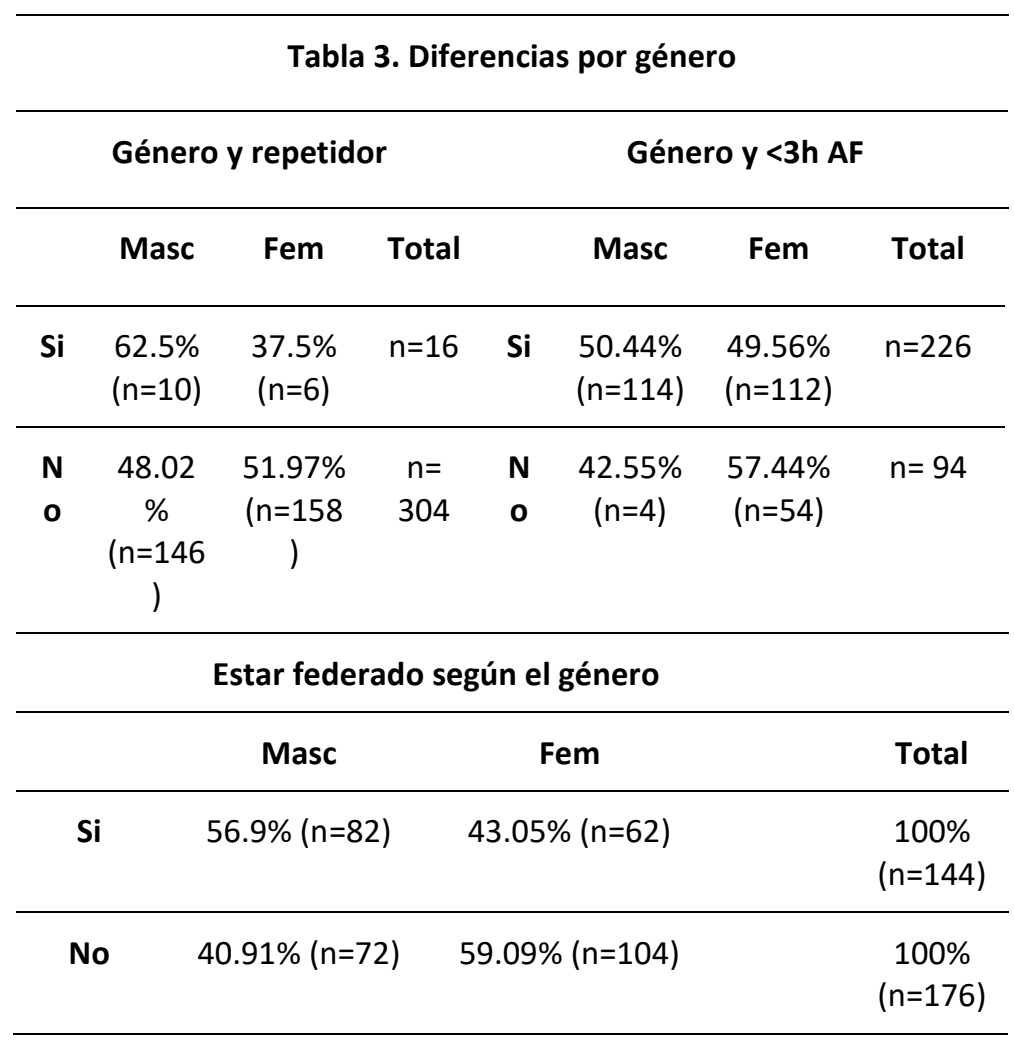

Los datos relativos a los niveles de resiliencia según el género aparecen representados en la tabla 4. Se muestran diferencias estadísticamente significativas a nivel $\mathrm{p}<0.05$ en la dimensión de persistencia, mostrando el sexo masculino valores medios más altos en todas las dimensiones, a excepción de la categoría de adaptabilidad donde las féminas obtuvieron valores medios más altos. 
Artículo Original. El rol de la resiliencia en la asociación entre la actividad física deportiva y aspectos académicos en escolares. Vol. 5, n. ${ }^{\text {o }}$ 3; p. 513-531, septiembre 2019. A Coruña. España ISSN 2386-8333.

Tabla 4. Niveles de Resiliencia según el género

\begin{tabular}{ccccc}
\hline & Masculino & Femenino & \\
\cline { 2 - 5 } & M (SD) & M (SD) & F & Sig \\
\hline Persistencia & $23.29(3.82)$ & $22.42(3.98)$ & 4.02 & $.046^{*}$ \\
\hline Control Bajo Presión & $19.92(4.39)$ & $19.69(4.21)$ & 0.21 & .643 \\
\hline Adaptabilidad & $14.87(2.55)$ & $14.94(2.92)$ & 0.05 & .821 \\
\hline Control y Propósito & $9.89(1.75)$ & $9.51(2.09)$ & 3.04 & .082 \\
\hline Espiritualidad & $5.80(1.41)$ & $5.84(1.22)$ & 0.25 & .616 \\
& & & & \\
\hline
\end{tabular}

El análisis de los niveles de Resiliencia en relación a las variables deportivas y académicas (Tabla 4), mostró asociaciones estadísticas para la dimensión de espiritualidad y la variable repetidor. En cuanto a las variables deportivas $\mathrm{AF}<3 \mathrm{H}$ se mostraron diferencias estadísticamente significativas a $\mathrm{p}<0.01$ en las dimensiones de adaptabilidad y control propósito y $\mathrm{p}<0.05$ en la categoría de persistencia. Asimismo, para la variable de estar federado se encontraron diferencias estadísticamente significativas a nivel 0.05 en las dimensiones de persistencia y control propósito y a nivel $\mathrm{p}<0.01$ en la categoría de adaptabilidad.

Tabla 5. Resiliencia y Repetidor, Act $<3 \mathrm{~h}$ y estar Federado

\begin{tabular}{|c|c|c|c|c|}
\hline \multicolumn{5}{|c|}{ Repetidor } \\
\hline & Sí & No & & \\
\hline & M (SD) & M (SD) & $\mathbf{F}$ & Sig \\
\hline Persistencia & $23.0(3.57)$ & $22.85(3.54)$ & 0.027 & .871 \\
\hline
\end{tabular}

Para citar este artículo utilice la siguiente referencia: Ramírez, I.A.; Alonso, J.M.; San Román, S.; Ubago, J.L.; Martínez, A.; Sánchez, M. ${ }^{a}$ (2019). El rol de la resiliencia en la asociación entre la actividad física deportiva y aspectos académicos en escolares. Sportis Sci J, 5 (3), 484 - 531 
Artículo Original. El rol de la resiliencia en la asociación entre la actividad física deportiva y aspectos académicos en escolares. Vol. 5, n. ${ }^{\text {o }}$ 3; p. 513-531, septiembre 2019. A Coruña. España ISSN 2386-8333.

\begin{tabular}{|c|c|c|c|c|}
\hline Control Bajo Presión & $21.75(.63)$ & $19.70(3.33)$ & 3.478 & .063 \\
\hline Adaptabilidad & $16.12(.60)$ & $14.84(1.82)$ & 3.334 & .069 \\
\hline Control y Propósito & $9.50(.56)$ & $9.71(2.73)$ & 0.178 & .673 \\
\hline \multirow[t]{4}{*}{ Espiritualidad } & $6.87(.59)$ & $9.70(1.31)$ & 10.54 & $.001 * *$ \\
\hline & \multicolumn{2}{|c|}{$\mathbf{A F}<\mathbf{3 H}$} & & \\
\hline & Sí & No & & \\
\hline & M (SD) & M (SD) & $\mathbf{F}$ & Sig \\
\hline Persistencia & $23.14(3.54)$ & $1.33(.56)$ & 4,475 & $.035 *$ \\
\hline Control Bajo Presión & $31.75(3.33)$ & $1.36(.54)$ & 1,167 & .281 \\
\hline Adaptabilidad & $16.12(1.82)$ & $1.21(.48)$ & 8,67 & $.003^{* *}$ \\
\hline Control y Propósito & $9.50(2.73)$ & $1.31(.49)$ & 5,79 & $.017 * *$ \\
\hline \multirow[t]{4}{*}{ Espiritualidad } & $6.87(1.31)$ & $1.59(.60)$ &, 242 & .623 \\
\hline & \multicolumn{2}{|c|}{ Estar federado } & & \\
\hline & Sí & No & & \\
\hline & M (SD) & M (SD) & $\mathbf{F}$ & Sig \\
\hline Persistencia & $23.58(3.56)$ & $22.23(3.92)$ & 4,475 & $.035^{*}$ \\
\hline Control Bajo Presión & $19.91(4.27)$ & $19.80(4.29)$ & 1,167 & .281 \\
\hline Adaptabilidad & $15.44(2.51)$ & $14.90(2.86)$ & 8,672 & $.003 * *$ \\
\hline Control y Propósito & 9.87 (1.83) & $9.70(1.94)$ & 5,792 & $.017 *$ \\
\hline Espiritualidad & $6.01(1.21)$ & $5.70(1.39)$ & 0,242 & .623 \\
\hline
\end{tabular}

\section{Discusión}

El presente estudio, realizado en escolares de tercer ciclo de Educación Primaria, tiene como objetivo definir las asociaciones existentes entre los niveles de Resiliencia y variables académicas, si el escolar ha repetido o no y variables físico deportivas, si los/as alumnos/as

Para citar este artículo utilice la siguiente referencia: Ramírez, I.A.; Alonso, J.M.; San Román, S.; Ubago, J.L.; Martínez, A.; Sánchez, M. ${ }^{a}$ (2019). El rol de la resiliencia en la asociación entre la actividad física deportiva y aspectos académicos en escolares. Sportis Sci J, 5 (3), 484 - 531 


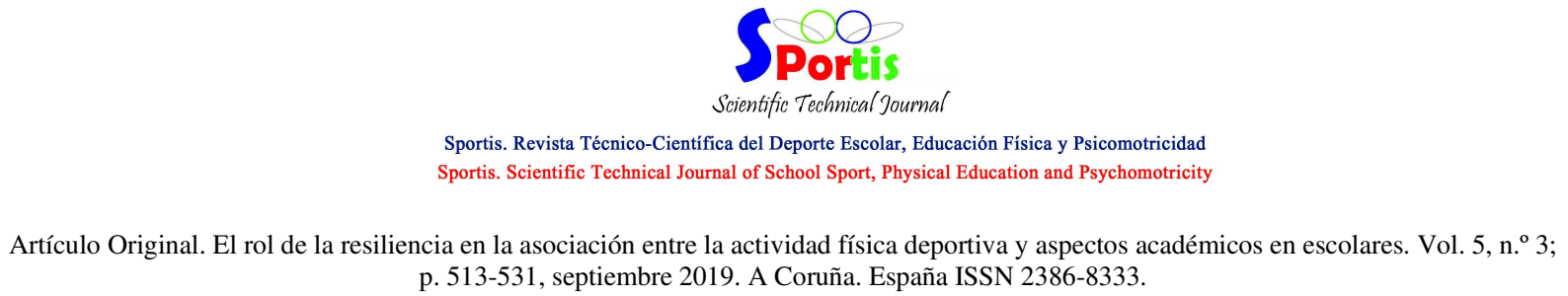
p. 513-531, septiembre 2019. A Coruña. España ISSN 2386-8333.

están federados y realizan más de tres horas de actividad física fuera del centro escolar. Estas finalidades radican en la importancia de conocer la influencia que ejerce la práctica deportiva hacia los niveles de Resiliencia, pues esta se relaciona directamente con el estar federado y los niveles de práctica de ejercicio. Algunos estudios de características similares son los llevados a cabo por Breton, Zurita y Cepero (2016), Hall (2011), Reche, Tuttle y Ortín (2014).

Revisando los descriptivos básicos, se estableció que la muestra estudiada estaba compuesta por escolares con una media de edad $(\mathrm{M}=11,6)$, donde el sexo masculino presenta mayor porcentaje que el femenino, destacando que siete de cada diez alumnos práctica más de tres horas de actividad física fuera del centro escolar datos que coinciden con los RamírezGranizo y Castro-Sánchez (2018), debiéndose esto a que cada vez son más actividades extraescolares a las que se encuentran apuntados los escolares en horario no lectivo, produciendo así resultados positivos ligados al bienestar psicosocial y física (Leventhal, et al., 2016). Asimismo, se puede ver que un poco más de la mitad de los sujetos no se encontraba federado en ninguna disciplina deportiva, esto puede deberse a que al encontrarse en una edad preadolescente los escolares pueden encontrarse apuntados a actividades extraescolares donde se les inicia en la competición y aún no han encontrado la motivación suficiente para apuntarse en una disciplina (Cantallops, Ponseti, Vidal, Antoni y Palou, 2012). Sin embargo, estos datos contradicen a lo que comenta Isorna, Rial y Vaquero-Cristóbal (2014), demostrando que ser miembro de algún club deportivo está fuertemente asociado con altos niveles de práctica de ejercicio físico, coincidiendo así con los resultados referentes a la frecuencia de actividad física estudiada. Del mismo modo se realizó una categorización de los niveles de Resiliencia, mostrando que los niveles con mayores puntuaciones son las de persistencia, control bajo presión y adaptabilidad, autores como Martin et al. (2014) o Newsome y Sullivan (2014), defienden estos resultados al trabajar el afrontamiento positivo ante la diversidad en diversos ámbitos. Por otro lado, la dimensión espiritual y control y propósito fueron las menos puntuadas, datos similares a los obtenidos por Reche et al. (2014).

$\mathrm{Si}$ atendemos a las diferencias por género, los niños realizan más ejercicio físico federado que las niñas, nuestros resultados son concordantes con los encontrados por Castro-Sánchez,

\footnotetext{
Para citar este artículo utilice la siguiente referencia: Ramírez, I.A.; Alonso, J.M.; San Román, S.; Ubago, J.L.; Martínez, A.; Sánchez, M. ${ }^{a}$ (2019). El rol de la resiliencia en la asociación entre la actividad física deportiva y aspectos académicos en escolares. Sportis Sci J, 5 (3), 484 - 531 DOI: https://doi.org/10.17979/sportis.2019.5.3.5603
} 


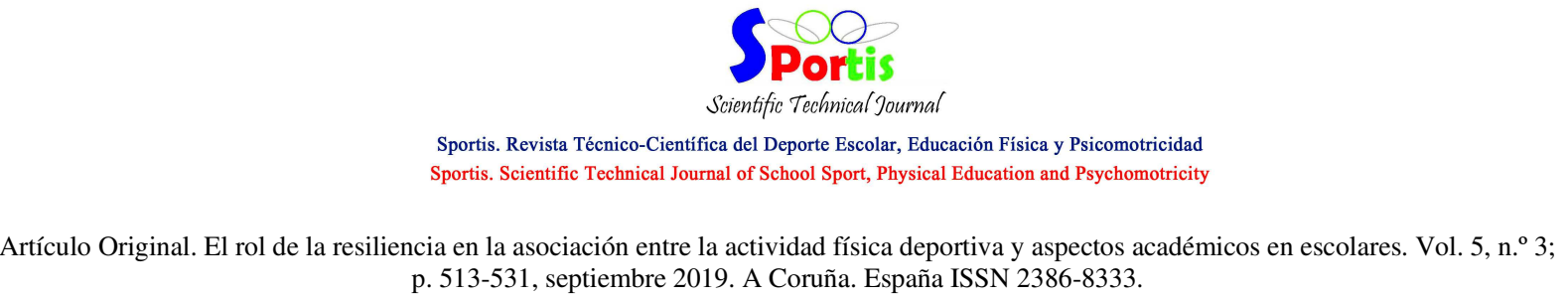
p. 513-531, septiembre 2019. A Coruña. España ISSN 2386-8333.

Zurita-Ortega, Martínez-Martínez, Chacón-Cuberos y Espejo-Garcés (2016), esto se puede deber a que los hombres mostraron valores más altos de motivación hacia la competición que las mujeres, habiendo diferencias entre ambos grupos. Asimismo, los niveles de resiliencia muestran diferencias significativas, los resultados arrojaron que el género masculino es más resiliente que el femenino a excepción de la categoría de adaptabilidad. Esto se debe a que existe una relación positiva entre la actividad física y los niveles de Resiliencia como indica Cho (2014), mostrando que los niños practican más actividad física que las niñas, en este caso ya que las niñas abandonan el ejercicio físico en edades tempranas en comparación a ellos. Sin embargo, estudios como los de Matalinares et al. (2011) o Ramírez-Granizo y CastroSánchez (2018), contradicen estos datos mostrando a las niñas más resilientes que ellos, ya que su disposición a adaptarse a situaciones estresantes es más elevada que la de ellos. De igual manera, diferentes estudios (Hawkins y Mulkey, 2005; Becoña, Minguez, López, Vàzquez y Lorenzo, 2006; López-Suárez, 2014) afirmaron no encontrar diferencias significativas entre el género y sus niveles de resiliencia.

Al analizar los resultados obtenidos de Resiliencia, variables académicas y actividad física, se encontraron diferencias estadísticamente significativas para la dimensión de espiritualidad y si ha repetido o no. En este caso podemos encontrar investigaciones como las de Reche, Tutte y Ortín (2014); Secades et al. (2014) y Zurita et al. (2016) donde confirman la relación que existe entre altos valores de Resiliencia y rendimiento deportivo, así como el rendimiento académico asociado a una de las dimensiones del autoconcepto. Los resultados muestran que aquellos sujetos con mayor nivel resiliente en la dimensión espiritual son los que no han repetido curso, esto se puede deber a que un nivel alto de Resiliencia promueve un bienestar emocional que produce un mejor control sistemático, bajos niveles de ansiedad y por lo tanto no afectaría a un desarrollo del nivel académico (Redondo-Elvira, Ibáñez-delPrado y Barbas-Abad, 2017).

De igual manera se encontró diferencias estadísticamente significativas en cuanto a la relación de practicar actividad física federada con las dimensiones de persistencia, control y propósito al igual que en adaptabilidad, datos que coinciden con estudios como los de

Para citar este artículo utilice la siguiente referencia: Ramírez, I.A.; Alonso, J.M.; San Román, S.; Ubago, J.L.; Martínez, A.; Sánchez, M. ${ }^{a}$ (2019). El rol de la resiliencia en la asociación entre la actividad física deportiva y aspectos académicos en escolares. Sportis Sci J, 5 (3), 484 - 531 DOI: https://doi.org/10.17979/sportis.2019.5.3.5603 


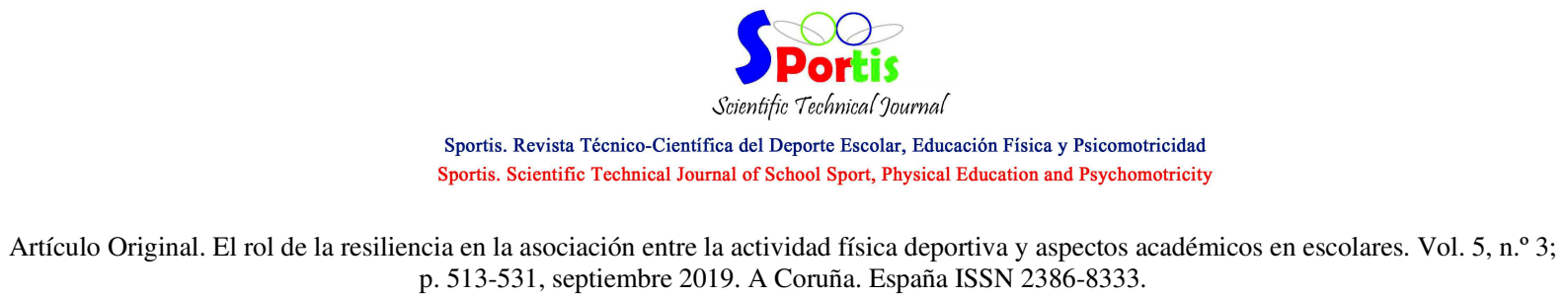
p. 513-531, septiembre 2019. A Coruña. España ISSN 2386-8333.

Almeida, Luciano, Lameiras y Buceta (2014); Petrie, Deiters y Harmison (2014) donde afirman que aquellos sujetos más deportistas poseían mayores estrategias para enfrentarse a situaciones desfavorables, superarlas de manera positiva afrontándolas desde la propia autoconfianza y factores externos como es el apoyo social que puede aportar un grupo o equipo federado. Esto coincide con Romero (2015) que señala que la práctica de actividad física y psicoeducativa mejora la asimilación de la Resiliencia favoreciendo el desarrollo integral de la persona, facilitando las diferentes transiciones de la vida de manera óptima gracias a una mejora de la fortaleza mental (Guillen y Laborde, 2014).

\section{Conclusiones}

Atendiendo a los resultados obtenidos, se señalan las siguientes conclusiones:

En torno a los niveles de resiliencia, las puntuaciones más elevadas se han dado en las dimensiones de persistencia, control bajo presión y adaptabilidad a los cambios. En cuanto a las diferencias por género, los niños mostraban capacidades resilientes más altas que las niñas en todas las categorías a excepción de la dimensión de adaptabilidad, donde ellas presentaban puntuaciones más altas.

Asimismo, aquellos participantes que no habían repetido curso presentan mejores niveles resilientes, al igual que los sujetos que estaban federados y practicaban más actividad física.

Por lo tanto, fomentar la actividad física y aumentar las capacidades resilientes tiene una relación positiva con el potencial de mejorar la salud, calidad de vida y el desarrollo de comportamientos saludables a nivel físico y mental en la etapa adulta.

\section{Referencias bibliográficas}

Almeida, P., Luciano, R., Lameiras, J., y Buceta, J. M. (2014). Beneficios percibidos de las lesiones deportivas: Estudio cualitativo en futbolistas profesionales y semiprofesionales. Revista de Psicología del Deporte, 23(2), 457-464.

\footnotetext{
Para citar este artículo utilice la siguiente referencia: Ramírez, I.A.; Alonso, J.M.; San Román, S.; Ubago, J.L.; Martínez, A.; Sánchez, M. ${ }^{a}$ (2019). El rol de la resiliencia en la asociación entre la actividad física deportiva y aspectos académicos en escolares. Sportis Sci J, 5 (3), 484 - 531 


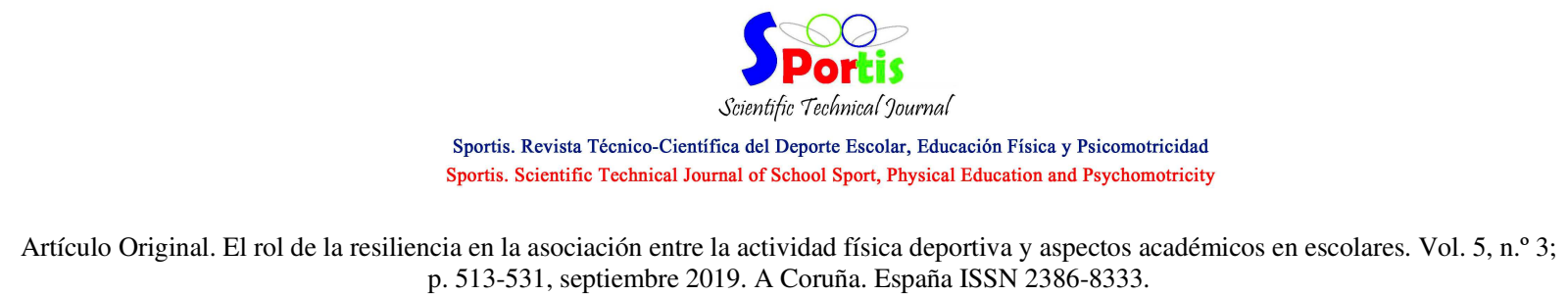
p. 513-531, septiembre 2019. A Coruña. España ISSN 2386-8333.

Becoña, E., Mínguez, M. C., López, A., Vázquez, M. J., y Lorenzo, M. C. (2006). Resiliencia y consumo de alcohol en jóvenes. Revista Salud y Drogas, 6(1), 89-111.

Branscum, P., y Crowson, H. M. (2016). The association between environmental and psychosocial factors towards physical activity and screen time of children: an application of the Integrative. Behavioural Model. Journal of Sports Sciences, 35(10), 982-988. https://doi.org/10.1080/02640414.2016.1206666

Bretón, S., Zurita, F., y Cepero, M. (2016). La Resiliencia como factor determinante en el rendimiento deportivo. Revisión Bibliográfica. E-balonmano.com: Revista de Ciencias del Deporte, 12(2), 79-88.

Bretón-Prats, S., Zurita-Ortega, F., y Cepero-González, M. (2017). Análisis de los constructos de autoconcepto y resiliencia, en jugadoras de baloncesto de categoría cadete. Revista de psicología del deporte, 26(S1), 127-132.

Broche, Y., Diago, C. y Herrera, L. F. (2012). Características resilientes en jóvenes deportistas y sus pares de la Universidad central Marta Abreu de las Villas. Revista de la Facultad de Psicología Universidad Cooperativa de Colombia, 8(14), 10-18.

Cabanyes-Truffino, J. (2010). Resiliencia: una aproximación al concepto. Revista de psiquiatría y Salud Mental, 3(4), 145-151. https://doi.org/10.1016/j.rpsm.2010.09.003

Castro-Sánchez, M., Chacón-Cuberos, R., Zurita, F., y Espejo, T. (2016). Niveles de resiliencia en base a modalidad, nivel y lesiones deportivas. Revista Retos, 29, 162165.

Chacón Cuberos, R.; Puertas Molero, P.; Pérez Cortés, A.J. (2017). Niveles de resiliencia según práctica de actividad física en estudiantes universitarios de Educación Física. ESHPA, 1(1): 59-67.

Chacón-Cuberos, R., Castro, M., Espejo, T. y Zurita, F., (2016). Estudio de la resiliencia en función de la modalidad deportiva: fútbol, balonmano y esquí. Revista Retos, 29, 157-161.

\footnotetext{
Para citar este artículo utilice la siguiente referencia: Ramírez, I.A.; Alonso, J.M.; San Román, S.; Ubago, J.L.; Martínez, A.; Sánchez, M. ${ }^{a}$ (2019). El rol de la resiliencia en la asociación entre la actividad física deportiva y aspectos académicos en escolares. Sportis Sci J, 5 (3), 484- 531 DOI: https://doi.org/10.17979/sportis.2019.5.3.5603
}

http://revistas.udc.es/ 


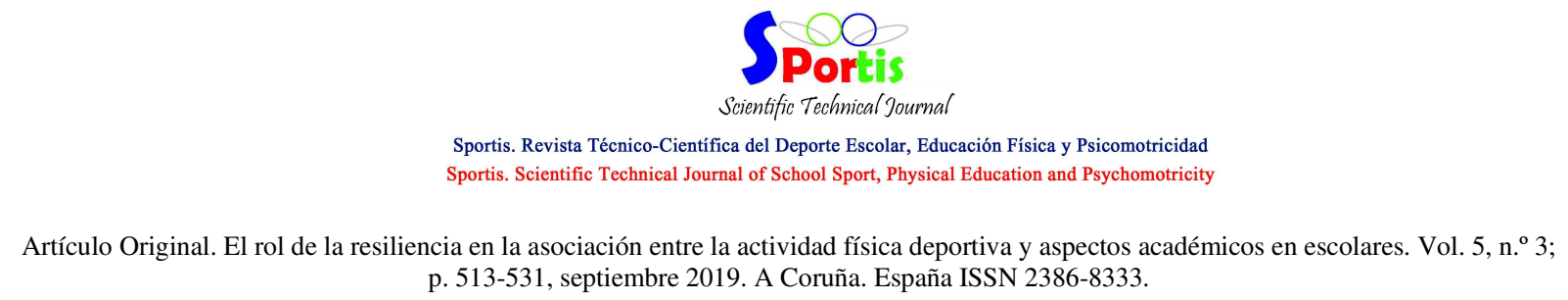

Cho, M. (2014). The influence of family's participation in recreational sports on its resilience and communication facilitation. Journal Exercise Rehabilitation, 10(5), 313318. https://doi.org/10.12965/jer.140161

Connor, K. M. y Davidson, J. R. (2003). Development of a new resilience scale: the Connor-Davidson Resilience Scale (CD-RISC). Depress Anxiety, 18, 76-82. https://doi.org/10.1002/da.10113

Espejo, T., Zurita, F., Chacón, R., Castro, M., Martínez-Martínez, A., y PérezCortés, A. J. (2018). Physical activity and self-concept: study two factors teens of rural area. Revista Iberoamericana de Psicología del Ejercicio y el Deporte, 13(2), 203-210.

Grotberg, E. H. (1995). A guide to promoting resilience in children: Strengthening the human spirit. The Hague, The Netherlands: Bernard Van Leer Foundation.

Guillén, F., y Laborde, S. (2014). Higher-order structure of mental toughness and the analysis of latent mean differences between athletes from 34 disciplines and nonathletes. Personality and Individual Differences, 60, 30-35. http://dx.doi.org/10.1016/j.paid.2013.11.019

Hall, N. (2011). "Give it everything you got”. Resilience for young males trough sport. International Journal of Men's Health, 10(1), 65-81. https://doi.org/10.3149/jmh.1001.65

Hawkins, R., y Mulkey, L. (2005). Athletic investment and academic resilience in a sample of african females and males in the middle grades. Education and Urban Society, 38(1), 62-88. https://doi.org/10.1177/0013124505280025

Isorna, M., Rial, A., y Vaquero-Cristóbal, R. (2014). Motivaciones para la práctica deportiva en escolares federados y no federados. Retos. Nuevas tendencias en Educación Física, Deporte y Recreación, 25, 80-84.

Jiménez-Beatty, J. E., Graupera, J. L., Martínez del Castillo, J., Martín, M., y Campos, A. (2007). Motivational factors and physicians' advice in physical activity in the older urban population. Journal of Aging and Physical Activity, 15(3), 241-256.

\footnotetext{
Para citar este artículo utilice la siguiente referencia: Ramírez, I.A.; Alonso, J.M.; San Román, S.; Ubago, J.L.; Martínez, A.; Sánchez, M. ${ }^{a}$ (2019). El rol de la resiliencia en la asociación entre la actividad física deportiva y aspectos académicos en escolares. Sportis Sci J, 5 (3), 484- 531 DOI: https://doi.org/10.17979/sportis.2019.5.3.5603
}

http://revistas.udc.es/ 


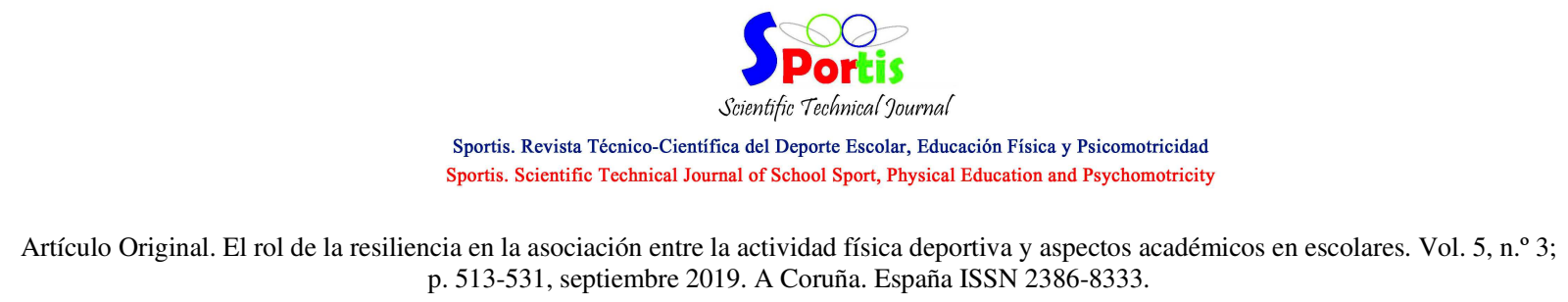
p. 513-531, septiembre 2019. A Coruña. España ISSN 2386-8333.

Kyle, T. L., Hernández, A., Reigal, R. E., y Morales, V. (2016). Efectos de la actividad física en el autoconcepto y la autoeficacia en preadolescentes. Retos. Nuevas tendencias en Educación Física, Deporte y Recreación, 29, 61-65.

Leventhal, K. S., DeMaria, L. M. Gillham, J. E., Andrew, G., Peabody, J. y Leventhal, S. M. (2016). A psychosocial resilience curriculum provides the "missing piece" to boost adolescent physical health: A randomized controlled trial of Girls First in India. Social Science \& Medicine, 161, 3746. https://doi.org/10.1016/j.socscimed.2016.05.004.

López-Suárez, M. (2014). Relación entre satisfacción con la vida y satisfacción con el deporte y en jóvenes deportistas. Tesis Doctoral: Universidad de las Palmas de Gran Canaria

Martin, L., Viljoen, M., Kidd, M., y Seedat, S. (2014). Are childhood trauma exposures predictive of anxiety sensitivity in school attending youth? Journal of Affective Disorders, 168, 5-12. https://doi.org/10.1016/j.jad.2014.06.035.

Matalinares, M. Arenas, C. Yaringaño, J. Sotelo, L. Sotelo, N. Díaz, G. Dioses, A. Ramos, R. Mendoza, P. Medina, Y. Pezua, M. Muratta, R. Pareja, C. y Tipacti, R. (2011). Factores personales de resiliencia y autoconcepto en estudiantes de primaria de Lima Metropolitana. Revista de Investigaciones Psicológicas, 14(1), 187-207. https://doi.org/10.15381/rinvp.v14i1.2083

Newsome, J. y Sullivan, C. J. (2014). Resilience and vulnerability in adolescents: Genetic influences on differential response to risk for delinquency. Journal Youth Adolescence, 43, 1080-1095. https://doi.org/10.1007/s10964-014-0108-9

Ortín-Montero, F. J., De la Vega, R. y Gosálvez-Botella, J. (2013). Optimismo, ansiedad-estado y autoconfianza en jóvenes jugadores de balonmano. Anales de psicología, 29, 637-641. http://dx.doi.org/10.6018/analesps.29.3.175751.

Ramírez-Granizo, I. A., y Castro-Sánchez, M. (2018). Análisis de los niveles de resiliencia en función del género y factores del ámbito educativo en escolares. ESHPA Education, Sport, Health and Physical Activity, 2(1), 50-61.

Para citar este artículo utilice la siguiente referencia: Ramírez, I.A.; Alonso, J.M.; San Román, S.; Ubago, J.L.; Martínez, A.; Sánchez, M. ${ }^{a}$ (2019). El rol de la resiliencia en la asociación entre la actividad física deportiva y aspectos académicos en escolares. Sportis Sci J, 5 (3), 484- 531 DOI: https://doi.org/10.17979/sportis.2019.5.3.5603 


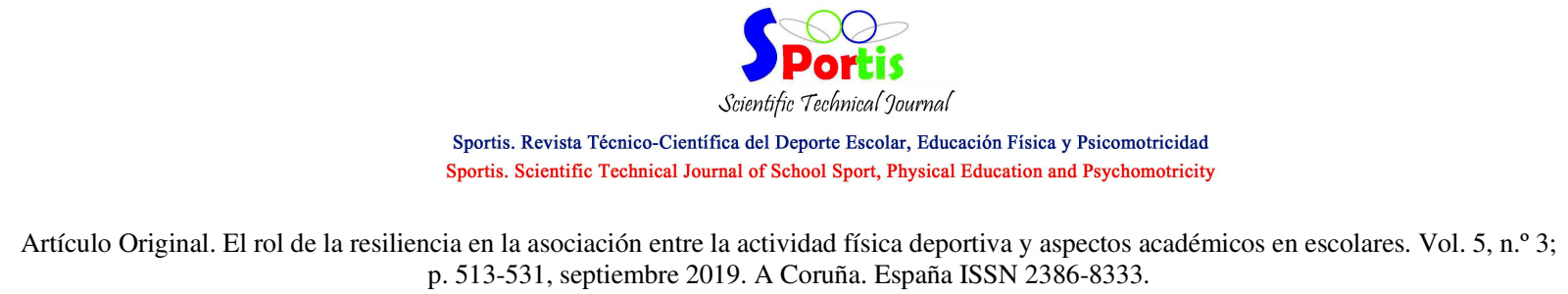
p. 513-531, septiembre 2019. A Coruña. España ISSN 2386-8333.

Ramírez-Granizo, I.A., Valdivia-Moral, P., Zurita-Ortega, F., \& Ubago-Jiménez, J.L. (2018). Relational research on sport practice and use of video games according to gender of schoolchildren from Granada. Journal of Human Sport and Exercise, 13(2proc), S495-S504. https://doi.org/10.14198/jhse.2018.13.Proc2.33

Reche, C., Tutte, V., y Ortín, F. J. (2014). Resiliencia, optimismo y burnout en judokas de competición uruguayos. Revista Iberoamericana de Psicología del Ejercicio y el Deporte, 9(2), 267-279.

Redondo-Elvira, T., Ibañez-del-Prado, C., y Barbas-Abad, S. (2017). Spiritually resilient. Relationship between spirituality and resilience in palliative care. Clinica $y$ Salud, 28, 117-121. https://doi.org/10.1016/j.clysa.2017.09.001.

Romero, C. (2009). Relación entre resiliencia, práctica de actividades recreativas físicas y consumo de alcohol en colegiales. Revista Digital de Educación Física, 5(26), $59-72$.

Rutter, M. (1993). Resilience: Some conceptual considerations. Journal of Adolescent Health, 14(8), 626-631. https://doi.org/10.1016/1054-139X(93)90196-V

Secades, X., Molinero, O., Barquín, R., Salguero, A., De la Vega, R., y Márquez, S. (2014). La resiliencia en el deporte: fundamentos teóricos, instrumentos de evaluación y revisión de la literatura. Cuadernos de Psicología del Deporte, 14(3), 83-92.

Zurita, F., Castro, M., Linares, M., y Chacón, R. (2017). Resiliencia, un elemento de prevención en actividad física. Sportis Sci J, 3(1), 50-62.

Zurita, F., Espejo, T., Cofré, c., Martínez, A., Castro, M., y Chacón, R. (2016). Influencia de la actividad física sobre la resiliencia en adultos con dolor de hombro. SPORT TK-Revista EuroAmericana de Ciencias del Deporte, 5(2), 53-58. https://doi.org/10.17979/sportis.2017.3.1.1726

Zurita, F., Fernández, R., Cepero, M., Zagalaz, M.L., Valverde, M., y Ramírez, P. (2009). The relationship between pain and physical activity in older adults that begin a

\footnotetext{
Para citar este artículo utilice la siguiente referencia: Ramírez, I.A.; Alonso, J.M.; San Román, S.; Ubago, J.L.; Martínez, A.; Sánchez, M. ${ }^{a}$ (2019). El rol de la resiliencia en la asociación entre la actividad física deportiva y aspectos académicos en escolares. Sportis Sci J, 5 (3), 484- 531 DOI: https://doi.org/10.17979/sportis.2019.5.3.5603
}

http://revistas.udc.es/ 
Artículo Original. El rol de la resiliencia en la asociación entre la actividad física deportiva y aspectos académicos en escolares. Vol. 5, n. ${ }^{\circ} 3$; p. 513-531, septiembre 2019. A Coruña. España ISSN 2386-8333.

program of physical activity. Journal of Human and Sport, 4(3), 284-297. https://doi.org/10.4100/jhse.2009.43.10 